\title{
Diurnal variation of pulmonary artery pressure in chronic heart failure
}

\author{
J SIMON R GIBBS, $†$ DAVID CUNNINGHAM, * LEONARD M SHAPIRO, \\ AMANDA PARK, * PHILIP A POOLE-WILSON, $\dagger$ KIM M FOX* \\ From the ${ }^{\star}$ National Heart Hospital, and the $\uparrow$ National Heart and Lung Institute, London
}

SUMMARY Variation in pulmonary artery pressure has important consequences for the interpretation of isolated pressure measurements in patients with chronic heart failure. To investigate the nature of diurnal variation in pulmonary artery pressure in chronic heart failure, eight angina-free men (aged 50-72 years) with treated chronic heart failure caused by ischaemic heart disease underwent continuous ambulatory pulmonary artery pressure recording by a transducer tipped catheter. The mean (1 SD) daytime pulmonary artery pressure was $29.6(5.0) \mathrm{mm} \mathrm{Hg}$ systolic and $13.7(5.6) \mathrm{mm} \mathrm{Hg}$ diastolic. The mean change in pressure from day to night was $+5 \cdot 1(3 \cdot 2)$ $\mathrm{mm} \mathrm{Hg}$ systolic and $+3 \cdot 8(1 \cdot 7) \mathrm{mm} \mathrm{Hg}$ diastolic; and the mean change from standing to lying $+9 \cdot 3$ $(2 \cdot 3) \mathrm{mm} \mathrm{Hg}$ systolic and $+6 \cdot 4(2 \cdot 1) \mathrm{mm} \mathrm{Hg}$ diastolic. In six of the eight patients there was considerable rise in pulmonary artery pressure at night, but in the two patients with the most severe symptoms there was no nocturnal rise.

In patients with chronic heart failure, nocturnal pulmonary artery pressure is not determined by postural change alone. But interpretation of isolated pulmonary artery pressure measurements must take the posture of the patient into account.

The measurement of central haemodynamic function on a single occasion in patients with chronic heart failure may be affected by the time of day. Haemodynamic changes that could be interpreted as being advantageous in patients with chronic heart failure have been shown on placebo treatment,${ }^{1}$ and after meals. ${ }^{2}$ This issue was highlighted when invasive studies showed an improvement in haemodynamic function over 24 hours without any therapeutic intervention. ${ }^{3}$

One possible explanation for these observations is that there is diurnal variation of central haemodynamic function that is confounding their interpretation. We investigated this hypothesis by measuring changes in pulmonary artery pressure in ambulant patients with chronic heart failure over a 24 hour period.

Requests for reprints to Dr J Simon R Gibbs, National Heart Hospital, Westmoreland Street, London W1M 8BA.

Accepted for publication 14 February 1989

\section{Patients and methods}

PATIENTS

Eight men (mean age 62; range 50-72) with chronic heart failure caused by ischaemic heart disease underwent continuous ambulatory pulmonary artery pressure measurement. They had been admitted to hospital for treatment of heart failure. All had undergone cardiac catheterisation and angiography on a previous hospital admission.

Their principal complaint was breathlessness on exertion and to a lesser extent tiredness. None of the patients had peripheral oedema, angina, or evidence of myocardial ischaemia on the electrocardiogram during exercise. The table shows details of individual patients. Three patients had previously undergone coronary artery surgery (cases 1,5 , and 7). Patients 1 and 6 were taking captopril $25 \mathrm{mg}$ three times a day, and the other patients were taking a dose of amiloride ( $5 \mathrm{mg}$ or $10 \mathrm{mg} /$ day). None of the patients was being treated with antiarrhythmic drugs, nitrates, calcium antagonists, non-steroidal anti-inflammatory agents, steroids, or anticoagulants. 
Table Data on patients

\begin{tabular}{|c|c|c|c|c|c|c|c|}
\hline Patient & Age & NYHA class & $\mathrm{VO}_{2} \max$ & Rest $L V E F$ & Exercise $L V E F$ & Pillows at night & Frusemide dose ( $m g$ ) \\
\hline $\begin{array}{l}1 \\
2 \\
3 \\
4 \\
5 \\
6 \\
7 \\
8\end{array}$ & $\begin{array}{l}72 \\
64 \\
60 \\
60 \\
68 \\
50 \\
61 \\
64\end{array}$ & $\begin{array}{l}2 \\
3 \\
3 \\
1 \\
2 \\
2 \\
3 \\
3\end{array}$ & $\begin{array}{l}12 \cdot 8 \\
11 \cdot 2 \\
14.0 \\
15 \cdot 3 \\
11 \cdot 3 \\
14.6 \\
10.4 \\
11.5\end{array}$ & $\begin{array}{l}45 \\
17 \\
25 \\
11 \\
31 \\
14 \\
14 \\
16\end{array}$ & $\begin{array}{l}42 \\
16 \\
15 \\
\\
18 \\
12 \\
16\end{array}$ & $\begin{array}{l}2 \\
2 \\
5 \\
2 \\
4 \\
3 \\
2 \\
5\end{array}$ & $\begin{array}{l}80 \times 1 \\
80 \times 2 \\
80 \times 1 \\
80 \times 1 \\
40 \times 1 \\
40 \times 1 \\
80 \times 2 \\
80 \times 2\end{array}$ \\
\hline
\end{tabular}

NYHA, New York Heart Association; $\mathrm{Vo}_{2}$ max, maximum rate of oxygen consumption on exercise (ml $\left./ \mathrm{kg} / \mathrm{min}\right) ; \mathrm{LVEF}, \%$ radionuclide left ventricular ejection fraction.

All of the patients had a cardiothoracic ratio of $>0.5$ without evidence of pulmonary oedema on a posterior-anterior chest radiograph. They had a normal forced expiratory volume and a normal vital capacity. The mean ( $1 \mathrm{SD})$ radionuclide left ventricular ejection fraction for the group was 21.6 $(10.7) \%$ at rest and $21.0(12.6) \%$ on exercise (data from patients 1 and 7 were not available on exercise). The mean right ventricular ejection fraction was $25 \cdot 1$ $(8.3) \%$ at rest and $24.3(10 \cdot 8) \%$ on exercise. The mean maximum rate of oxygen consumption on exercise was $12 \cdot 6(1 \cdot 7) \% \mathrm{ml} / \mathrm{kg} / \mathrm{min}$.

\section{PRESSURE MEASUREMENT AND RECORDING}

Pulmonary artery pressure was recorded by a $6 \mathrm{~F}$ micromanometer-tipped catheter (Gaeltec). The signal was amplified with a portable battery driven amplifier and a frequency modulated signal was recorded on tape. In patients 1,5 , and 6 recordings were made on an Oxford Medilog II miniature cassette recorder that was modified by the insertion of a frequency modulator. In patients $2,3,4,7$, and 8 recordings were made on a Hewlett Packard 3964A Instrumentation Recorder to which the patient was attached by a $7 \mathrm{~m}$ coaxial cable.

The catheter and amplifier had a linear response to pressure over the range 0 to $225 \mathrm{~mm} \mathrm{Hg}(-150 \mathrm{mV}$ to $+500 \mathrm{mV}$ ) with a correlation coefficient of 0.9997 . The frequency response of the catheter plus amplifier was flat from 0 to $14 \mathrm{~Hz}(-3 \mathrm{~dB})$. The frequency response of the recording and playback equipment was higher than this. The sensitivity of the catheter was $5 \mu \mathrm{V} / \mathrm{V} / \mathrm{mm} \mathrm{Hg}$ and the maximum hysteresis error was $0.1 \%$ of full scale. The temperature coefficient of sensitivity was $-0.06 \% /{ }^{\circ} \mathrm{C}$ and of zero was $0.01 \%$ of full scale. Zero drift of the catheter, amplifier, and recorders was less than $2 \%$ of full scale over 24 hours.

After being sterilised in Cidex (Surgikos), the catheter was washed and soaked in physiological saline. Immediately before we inserted the catheter into a patient, we calibrated the system by sealing the catheter tip in a sterile plastic cylinder that was attached via a sterile tube to a 239P air-operated dead-weight pressure balance (Budenberg Gauge Company). Static pressure calibrations were recorded on tape and read off a voltmeter at $0,1 \cdot 5,2 \cdot 5$, $5 \cdot 0,7 \cdot 5$, and $10.0 \mathrm{kPa}(1 \mathrm{kPa}=7.5006 \mathrm{~mm} \mathrm{Hg})$. This calibration procedure was repeated immediately after the catheter was removed from a patient and the calibration lines were used to confirm the absence of a change in catheter sensitivity and to extrapolate the pulmonary artery pressures from the recordings.

\section{STATISTICAL ANALYSIS}

The tape recordings of pulmonary artery pressure were replayed on to an ink jet chart recorder (Siemens-Elema Mingograf 82). The systolic and diastolic pressures were sampled every 12 minutes and plotted on a graph (figs 1 and 2). To take account of respiratory variation, the mean systolic and diastolic pressures were determined manually over a 30 second period.

The 24 hour period was divided into day and night for each individual according to the time at which they recorded retiring to bed in the evening and getting out of bed the following morning. The systolic and diastolic pressures were averaged for these periods and compared by a Student's paired $t$ test.

\section{PROTOCOL}

The patients gave written informed consent to this procedure, which was approved by the National Heart and Chest Hospitals ethics committee.

For the day preceding catheterisation and during the study the patients kept a diary of their activities. The severity of any symptoms experienced was recorded on a visual analogue scale.

The patients fasted for four hours before catheterisation. No premedication was given. In the catheterisation laboratory a $7 \mathrm{~F}$ introducer was inserted into a subclavian vein under local anaesthetic and through this the catheter was inserted and located, under $x$ ray screening, in either the proximal left or right pulmonary artery. The introducer was then withdrawn 


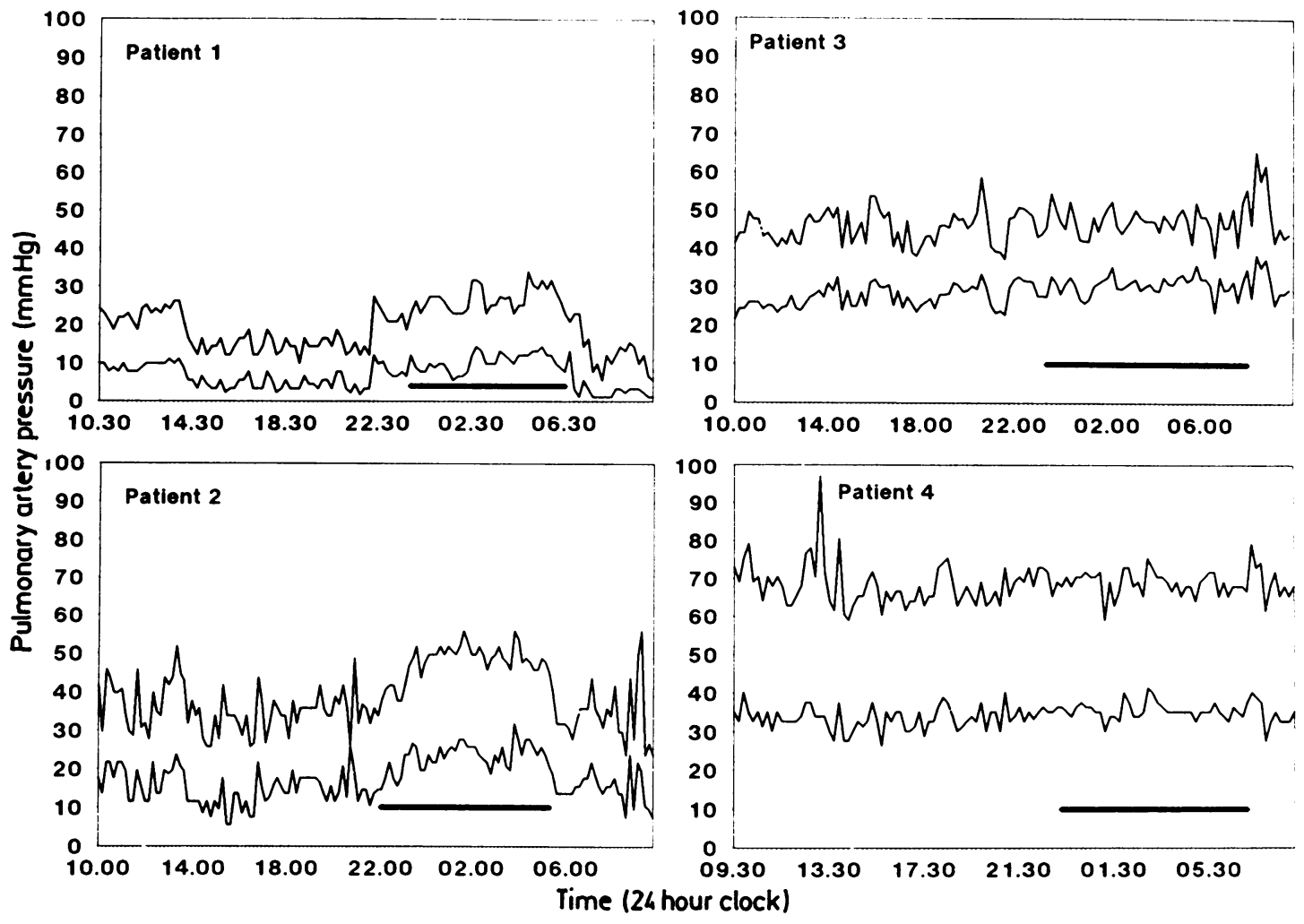

Fig 1 Twenty four hour recordings of systolic and diastolic pulmonary artery pressure in patients 1 to 4 . The time the patient was in bed overnight is indicated by a thick line.

and the catheter was stitched in place to the skin. Pressure recording was started within 30 minutes of catheter insertion.

On their return to the general ward the patients were fed and those patients catheterised in the morning took their diuretics. The patients on twice daily diuretics received their second dose at $14.00 \mathrm{~h}$. The two patients on captopril took the first dose with their diuretics, the second dose at $14.00 \mathrm{~h}$, and the third dose at $22.00 \mathrm{~h}$. On the second and subsequent days the first dose of drugs was administered at $\mathbf{0 8 . 0 0}$ h. The patients were encouraged to go about their normal daily activities within the confines of the ward and adjacent staircase. Two organised activities were performed between $14.30 \mathrm{~h}$ and $16.00 \mathrm{~h}$ during the afternoon of the day of catheterisation. These were a period of recording the postural changes in pressure followed by a walk on the treadmill at a comfortable walking pace for the patient. Pressure was recorded with the patient in different postures for 10 minutes each: lying down on a bed with one pillow, sitting on the edge of the bed, and standing up.

The electrocardiogram was recorded simultan- eously with a Oxford Medilog II frequency modulated recorder to screen for arrhythmias and ST segment changes during the period of pressure recording.

\section{Results}

All but one of the patients underwent catheterisation in the morning. In patients 1 to 4 the systolic and diastolic pulmonary artery pressures were recorded for 24 hours (fig 1 ) and in patients 5 to 8 for 48 hours (fig 2).

For the whole group the mean daytime systolic pulmonary artery pressure was $29.6(5.0) \mathrm{mm} \mathrm{Hg}$ and diastolic pressure was $13.7(5 \cdot 6) \mathrm{mm} \mathrm{Hg}$. During the night the systolic pressure rose to $36.0(4 \cdot 1)$ $\mathrm{mm} \mathrm{Hg}(\mathrm{p}<0.005)$ and the diastolic to $17.6(2.9)$ $\mathrm{mm} \mathrm{Hg}(\mathrm{p}<0.001)$. The mean increase in systolic pressure from day to night was $5 \cdot 1(3 \cdot 2) \mathrm{mm} \mathrm{Hg}$ and from standing to lying was $9.3(2.3) \mathrm{mm} \mathrm{Hg}$ $(p<0.025)$. The mean increase in diastolic pressure from day to night was $3.8(1.7) \mathrm{mm} \mathrm{Hg}$ and from standing to lying was $6.4(2.1) \mathrm{mm} \mathrm{Hg}(\mathrm{p}<0.025)$. 


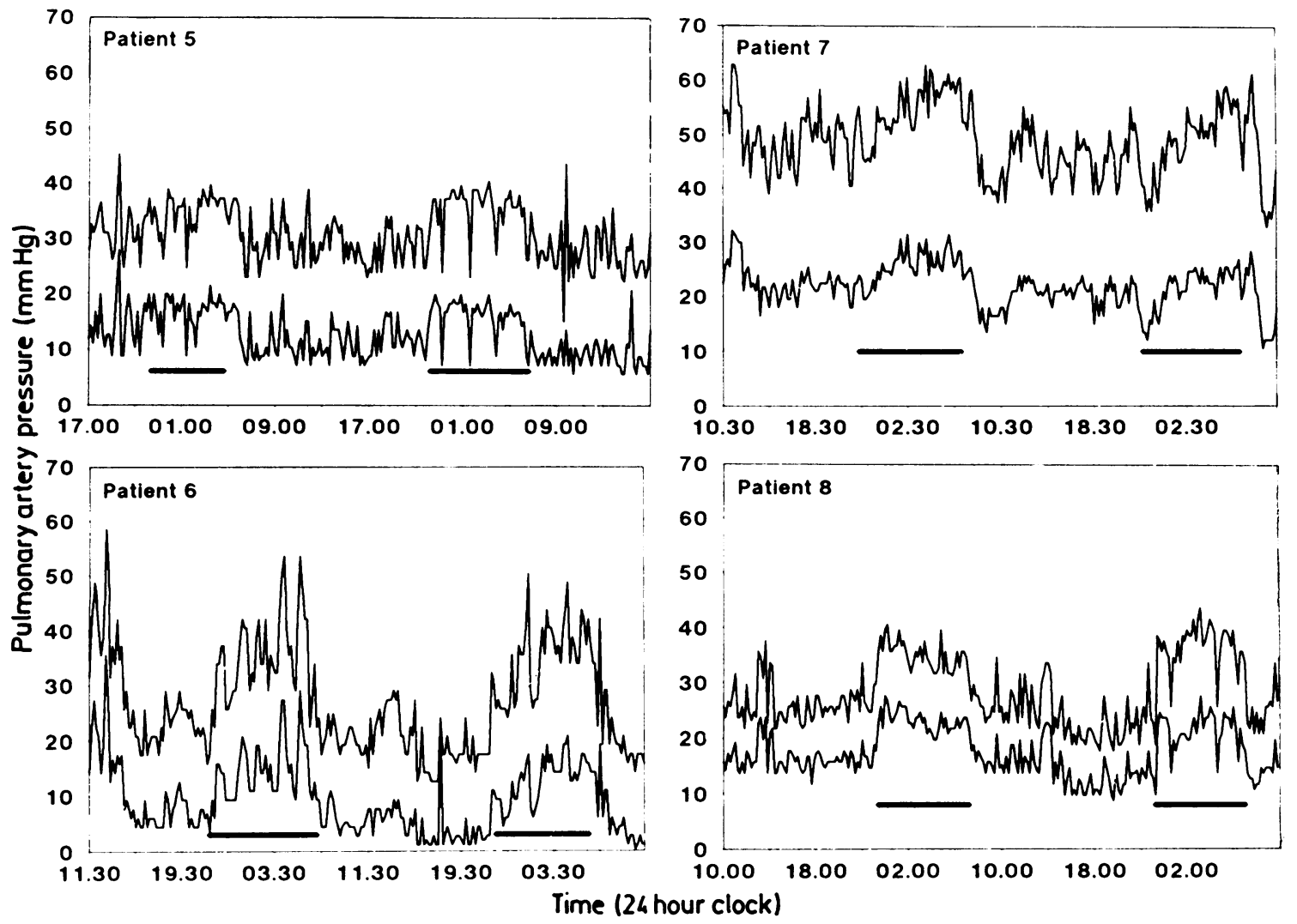

Fig 2 Forty eight hour recordings of systolic and diastolic pulmonary artery pressure in patients 5 to 8. The time the patient was in bed overnight is indicated by a thick line.

In six of the eight patients there was considerable diurnal variation in pulmonary artery pressure. In four of these patients recordings were obtained over 48 hours and the diurnal variation in the first 24 hours was similar to that in the second 24 hours. In two of the eight patients (cases 3 and 4) there was no pressure rise during the night. These patients had the worst symptoms, though they had no other features that distinguished them from those whose pulmonary artery pressure showed diurnal variation.

One possible explanation for the nocturnal rise in pressure might be postural change. Comparison of the changes in systolic and diastolic pressure from day to night and lying to standing (figs 3 and 4 ) shows a change in the same direction with a small but statistically significant difference in the magnitude of the day to night and lying to standing changes. No correlation was found in individuals between postural pressure changes and nocturnal increases in either systolic or diastolic pressure. There was also no correlation between the nocturnal rise in pressure and the number of pillows with which patients slept (table).

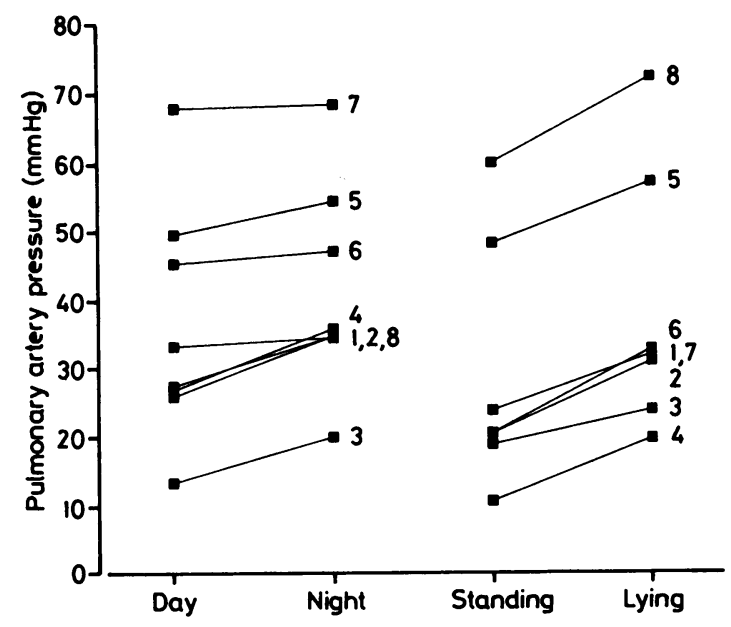

Fig 3 Comparison of day to night changes in systolic pulmonary artery pressure with changes recorded from standing to lying during the afternoon. Individual patients are numbered. 
34

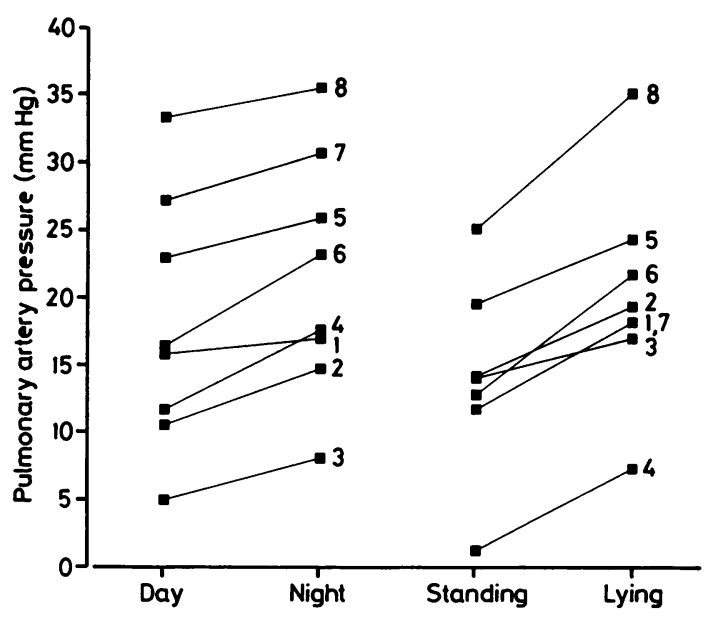

Fig 4 Comparison of day to night changes in diastolic pulmonary artery pressure with changes recorded from standing to lying during the afternoon. Individual patients are numbered.

Monitoring of the electrocardiogram showed no ST segment changes and no sustained rhythm disturbance. During the period of recording the patients noted few episodes of breathlessness or tiredness: these were rated as mild. Apart from slight discomfort at the site of catheter insertion, which did not restrict the activity of the patients, there were no complications from the catheterisation procedure.

\section{Discussion}

We have shown a considerable variability in pulmonary artery pressure throughout the day: six out of the eight patients showed a substantial rise in pulmonary artery pressure while they were in bed at night. Despite the invasive nature of the investigation, the patients stated they were almost as mobile as they were at home and all reported sleeping well. For the whole group the mean pulmonary artery systolic and diastolic pressures at rest were within normal limits during the day but became abnormal at night. In the $48 \mathrm{~h}$ recordings the same pattern of pressure rise was reproduced each night. Pulmonary artery pressure may be affected by left ventricular function, ${ }^{45}$ exercise, effects of diuretics, ${ }^{6}$ meals, ${ }^{27}$ respiratory pattern, ${ }^{8-10}$ autonomic effects such as fear, ${ }^{11-13}$ and temperature. ${ }^{14}$ It seems that the previous inconsistences in the assessment of pulmonary artery pressure could be explained by these effects together with diurnal and postural changes. ${ }^{15-17}$ The effect of drugs on the behaviour of the pulmonary artery pressure in our patients cannot be quantified, but it is likely'that they
Gibbs, Cunningham, Shapiro, Park, Poole-Wilson, Fox

are partially responsible for the variability that we saw.

The use of micromanometer tipped catheters for extended pressure measurement overcomes many of the problems inherent in fluid-filled systems such as errors in transducer balancing, calibration and levelling to the zero reference point, as well as artefacts such as catheter whip, catheter impact, and end pressure. ${ }^{18}$ Micromanometer catheters have their own shortcomings, in particular, zero drift, temperature sensitivity, and fragility. The studies in our eight patients did not seem to be hampered by these problems.

There is only limited information about normal diurnal variation of pulmonary artery pressure. In ten normal individuals who underwent polygraphic monitoring pulmonary artery pressure rose significantly, from an average of $18 / 8 \mathrm{~mm} \mathrm{Hg}$ while awake to $23 / 12 \mathrm{~mm} \mathrm{Hg}$ during sleep. ${ }^{19}$ Diurnal variation of pulmonary artery diastolic pressure in patients with chest pain showed a significant rise in pressure between midnight and $06.00 \mathrm{~h} .{ }^{20}$

The changes that we saw in pulmonary artery pressure in patients in bed at night are largely consistent with a change in posture. The discrepancy between standing to lying and day to night pressure changes may be explained either because the patients were propped up on more pillows at night than they were when they lay down during the day or because postural pressure changes themselves varied according to the time of day itself and to the timing of drug administration.

Two further observations, however, suggest that the nocturnal pressure rise cannot be explained entirely by posture. First, the rise in pressure at night was not always sudden and it varied considerably. In four of the patients (cases 1,2,6, and 7) the nocturnal rise in pressure was of gradual onset and tended to increase during the night. This is in contrast with the postural pressure changes during the day which took less than 15 seconds to equilibrate at their new value and then remained steady. Second, two patients had no nocturnal rise in pressure. Their posture at night did not differ from that of the other patients and they had similar postural pressure changes during the day compared with the rest of the group.

Sleep itself is probably not responsible for the pressure changes because hypnosis had no effect on cardiac output or pulmonary vascular pressures. ${ }^{21}$ In studies of neural control of pulmonary artery pressure in patients with heart failure treated with ganglionic and sympathetic blocking agents a fall in pulmonary artery pressure was recorded during sleep. ${ }^{22}$ This finding may have reflected the use or consumption of the drugs.

In patients with treated chronic heart failure, there 
is often diurnal variation of pulmonary artery pressure, the notable feature of which is a pronounced pressure rise at night. Superimposed upon this are other sources of temporary variation such as posture, alterations of which may cause large pressure changes. Although lying down may have been the principal cause of the nocturnal rise in pulmonary artery pressure seen in six of our eight patients, the pattern of a gradual rise in pressure in some of these patients and the lack of nocturnal rise in pressure in a further two patients suggest that other unexplained factors may influence the pulmonary artery pressure at night, and that different mechanisms may predominate in different individuals. Patients with chronic heart failure and a nocturnal increase in pulmonary artery pressure may be at increased risk of developing paroxysmal nocturnal dyspnoea.

JSRG is a British Heart Foundation Junior Research Fellow. We thank Gaeltec for the supply of the catheters.

\section{References}

1 Franciosa JA, Weber KT, Levine TB, et al. Hydralazine in the long-term treatment of chronic heart failure: lack of difference from placebo. $\mathrm{Am}$ Heart $J$ 1982;104:587-94.

2 Cornyn JW, Massie BM, Unverferth DV, Leier CV. Hemodynamic changes after meals and placebo treatment in chronic congestive heart failure. Am J Cardiol 1986;57:238-41.

3 Packer M, Medina N, Yushak M. Hemodynamic changes mimicking a vasodilator drug in the absence of drug therapy after right heart catheterization in patients with chronic heart failure. Circulation 1985; 71:761-6.

4 Scheinman M, Evans GT, Weiss A, Rapaport E. Relationship between pulmonary artery end-diastolic pressure and left ventricular filling pressure in patients in shock. Circulation 1973;47:317-24.

5 Kaltman AJ, Herbert WH, Conroy RJ, Kossman CE. The gradient in pressure across the pulmonary vascular bed during diastole. Circulation 1966;34: 377-84.

6 Dikshit K, Vyden JK, Forrester JS, Chatterjee K, Prakash R, Swan HJC. Renal and extrarenal hemodynamic effects of furosemide in congestive heart failure after acute myocardial infarction. $N$ Engl J Med 1973;288:1087-90.

7 Siemienczuk D, Greenberg B, Broudy D. Hemodynamic effects of eating in patients with congestive heart failure [Abstract]. Circulation 1984;70 (suppl II): II-306.

8 Coccagna G, Mantovani M, Brignani F, Parchi C, Lugaresi $\mathrm{E}$. Continuous recording of pulmonary and systemic arterial pressure during sleep in the syndrome of hypersomnia with periodic breathing. Bull Physio-path Resp 1972;8:1159-72.
9 Schroeder JS, Motta J, Guilleminault C. Hemodynamic studies in sleep-apnea. In: Guilleminault C, Dement WC, eds. Sleep-apnea syndrome (Kroc Foundation series vol. 11). New York: Alan R Liss, 1978:177-96.

10 Tilkian AG, Guilleminault C, Schroeder JS, Lehrman KL, Simmons FB, Dement WC. Hemodynamics in sleep-induced apnea. Studies during wakefulness and sleep. Ann Intern Med 1976;85:714-9.

11 Hickam JB, Cargill WH, Golden A. Cardiovascular reactions to emotional stimuli. Effect on the cardiac output, arteriovenous oxygen difference, arterial pressure, and peripheral resistance. J Clin Invest 1948;27:290-8.

12 Lewis BM, McElroy WT, Hayford-Wesling EJ, Samberg LC. The effects of body position, ganglionic blockade and norepinephrine on the pulmonary capillary bed. J Clin Invest 1960;39:1345-51.

13 Stead EA, Warren JV, Merrill AJ, Brannon ES. The cardiac output in male subjects as measured by the technique of right atrial catheterization. Normal values with observations on the effect of anxiety and tilting. J Clin Invest 1945;24:326-31.

14 Burch GE, Hyman A. Influence of hot and humid environment upon cardiac output and work in normals and in patients with chronic congestive heart failure at rest. Am Heart $J$ 1957;53:665-79.

15 Bevegard S, Holmgren A, Jonnson B. The effect of body position on the circulation at rest and during exercise, with special reference to the influence on stroke volume. Acta Physiol Scand 1960;49:279-98.

16 Donald KW, Bishop JM, Cumming G, Wade OL. The effect of nursing positions on the cardiac output in man. Clin Sci 1953;12:199-216.

17 Fournier P, Mensch-Dechene J, Ranson-Bitker B, Valladares W, Lockhart A. Effects of sitting up on pulmonary blood pressure, flow, and volume in man. J Appl Physiol 1979;46:36-40.

18 Grossman W. Pressure measurement. In: Grossman W, ed. Cardiac catheterization and angiocardiography. 3rd ed. Philadelphia: Lea and Febiger, 1986:118-34.

19 Lugaresi E, Coccagna G, Cirignotta F, et al. Breathing during sleep in man in normal and pathological conditions. In: Fitzgerald RS, Gautier H, Lahiri S, eds. The regulation of respiration during sleep and anesthesia. (Advances in experimental medicine and biology vol 99.) New York and London: Plenum Press, 1978:35-45.

20 Levy RD, Cunningham D, Shapiro LM, Wright C, Mockus L, Fox KM. Diurnal variation in left ventricular function: a study of patients with myocardial ischaemia, syndrome $\mathrm{X}$, and of normal controls. $\mathrm{Br}$ Heart J 1987;57:148-53.

21 Arvidsson $\mathrm{T}$, Astrom $\mathrm{H}$, Bevegard S, Jonsson $\mathrm{B}$. Circulatory effects of suggested leg exercise and fear induced under hypnotic state. Prog Resp Res 1970;5:365-74.

22 Halmágyi D, Felkai B, Iványi J, Zsótér T, Tényi $M$, Szücs Zs. The role of the nervous system in the maintenance of pulmonary arterial hypertension in heart failure. Br Heart $J$ 1953;15:15-24. 